\title{
Outputorientierte Betrachtung der nass-mechanischen Aufbereitung von polyolefinreichen Abfällen für das rohstoffliche Recycling
}

\author{
Lukas Kranzinger · Roland Pomberger · Markus Bauer · Markus Lehner · Daniel Schwabl · Helmut Flachberger • \\ Wolfgang Hofer
}

Online publiziert: 5. September 2017

(c) Der/die Autor(en) 2017. Dieser Artikel ist eine Open-Access-Publikation.

Zusammenfassung In den letzten Jahren wird immer häufiger davon berichtet, dass Kunststoffe mit ihren Inhaltsstoffen (Weichmacher, Flammschutzmittel etc.) ein Umweltrisiko darstellen. Dennoch wäre eine industrialisierte $\mathrm{Ge}$ sellschaft ohne diesen Werkstoff nicht mehr vorstellbar, denn die Anwendungsbereiche sind sehr vielfältig. Ein möglicher Ansatz, um diesem wachsenden Problem gegenzusteuern, ist die Implementierung einer flächendeckenden und funktionierenden Sammlung, die das Recycling der Altkunststoffe ermöglicht. Ein Ländervergleich zeigt, dass nur ein geringer Anteil der in Verkehr gesetzten Kunststoffmengen gesammelt und recycelt wird. Im Vergleich zu den Sammelmengen ist die Output-bezogene Recyclingrate, somit

DI L. Kranzinger $(\bowtie)$.

Univ.-Prof. DI Dr. R. Pomberger

Lehrstuhl für

Abfallverwertungstechnik

und Abfallwirtschaft,

Montanuniversität Leoben,

Franz-Josef-Straße 18, 8700 Leoben,

Österreich

lukas.kranzinger@unileoben.ac.at

DI Dr. M. Bauer .

Univ.-Prof. DI Dr. M. Lehner Lehrstuhl für Verfahrenstechnik des industriellen Umweltschutzes, Montanuniversität Leoben,

Franz-Josef-Straße 18, 8700 Leoben, Österreich

DI D. Schwabl

Univ.-Prof. DI Dr. H. Flachberger Lehrstuhl für Aufbereitung und Veredlung, Montanuniversität Leoben, Franz-Josef-Straße 18, 8700 Leoben, Österreich

DDI W. Hofer

OMV Refining \& Marketing $\mathrm{GmbH}$,

Trabrennstraße 6-8, 1020 Wien,

Österreich die tatsächliche stoffliche Verwertung, derzeit noch bescheiden.

Die Untersuchungen des vorliegenden Forschungsprojekts „Plastic Reborn" befassen sich einerseits mit der Identifikation und andererseits mit der Darstellung der Entsorgungswege von polyolefinreichen Abfallströmen in der österreichischen Abfallwirtschaft (Abfallmengenflussbild). Darüber hinaus werden die Verwertungspotenziale für die aus der nass-mechanischen Aufbereitung generierten Outputfraktionen bestimmt. Das Abfallmengenflussbild zeigt, dass trotz einer gut funktionierenden getrennten Sammlung, noch ein großes ungenutztes Polyolefinpotenzial für das Recycling verfügbar ist. Die ersten Trennversuche mit einer nassmechanischen Aufbereitung haben gezeigt, dass die Polyolefine aus den Abfallströmen abgetrennt werden können. Die Kunststoffmehrmenge kann ein signifikanter Beitrag zur Umsetzung der Strategie zur europäischen Kreislaufwirtschaft sein.

Schlüsselwörter Polyolefine $\cdot$ Nassmechanische Aufbereitung . Kunststoffrecycling

\section{Output Oriented Analysis of the Wet Mechanical Processing of Polyolefin-rich Waste for Feedstock Recycling}

Abstract In the last few years reports
have become more and more frequent
that plastics and their constituents
(plasticiser, flame retardant, etc.) are
an environmental risk. Nevertheless,
an industrialised society without this
material would no longer be conceiv-
able, since the fields of application are
very diverse. One possible approach to
counteract this growing problem is the
implementation of a comprehensive
and functioning collection system that
allows recycling of post-consumer plas- tics. A comparison between countries shows that only a small portion of the plastic quantities put into circulation are collected and recycled. Compared to the collected quantities, the outputrelated recycling rate, which is the actual material recycling, is currently still modest.

The investigations of the present research project "Plastic Reborn" deal, on the one hand, with the identification and, on the other hand, with the depiction of the discharge paths of polyolefin-rich waste streams in Austrian waste management (waste flow diagram). In addition, exploitation potentials for the output fractions generated from the wet-mechanical processing are determined. The waste flow diagram shows that despite a wellfunctioning separate collection a large potential of unused polyolefin is still available for recycling. The first separation tests with a wet-mechanical processing have shown that the polyolefin potential of the waste streams can be separated. These excess quantities of plastics can be a significant contribution to the implementation of the European circular economy strategy.

Keywords Polyolefins · Wet mechanical processing $\cdot$ Plastics recycling

\section{Einleitung}

Im Jahr 2015 wurden weltweit 322 Mio. t (Millionen Tonnen) Kunststoffe produziert, Tendenz steigend. Davon kamen ca. 1,1 Mio. $t$ in Österreich zum Einsatz, wobei $40 \mathrm{M}-\%$ (Massenprozent) in der Verpackungsindustrie verwendet wurden. Hierbei kamen hauptsächlich Polyolefine (mengenmäßig vor allem Polyethylen (PE) und Polypropylen (PP)) zum Einsatz (Plastics 2017). Bei diesen Verpackungskunst- 


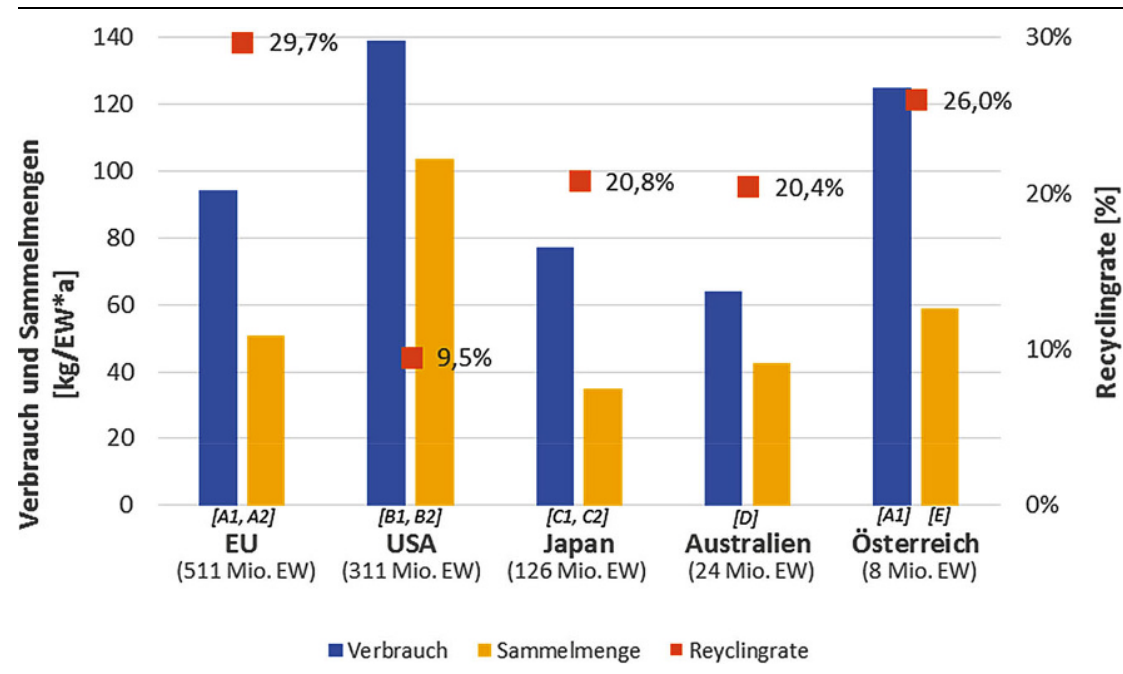

Abb. 1 Ländervergleich: Kunststoffverbrauch, -sammlung und -recyclingraten in Kilogramm pro Einwohner und Jahr [kg/EW*a]. (Quellen: A1 (Plastics 2015a), A2 (Plastics 2017), B1 (Plastic 2017), B2 (EPA2016), C1 (PWMI 2015), C2 (PWMI 2016), D (A'Vard \& Allan 2014), E(Plastics 2015b))

stoffen tritt das Ende des Produktlebenszyklus bereits nach sehr kurzer Verwendungsdauer (Tage bis Wochen) ein. Hingegen verlieren Kunststoffe aus anderen Verwendungsbranchen (z. B.: Bau- oder Elektronikbranche) ihre Funktion frühestens nach ein paar Jahren und lassen sich erst mit zeitlicher Verzögerung als Altkunststoff in der Abfallwirtschaft wiederfinden. Für eine flächendeckende Erfassung dieser Altkunststoffe werden weltweit bereits getrennte Sammelsysteme (Haus-zuHaus-Sammlung, Sammelpunkte oder Recyclinghöfe mit Monofraktions- bzw. Vermengt-Sammelbehältern) errichtet. Damit werden eine sortenreine Sammlung und das Recycling von Kunststoffen ermöglicht.

In Österreich umfasst der Zuweisungskatalog der getrennten Sammlung lediglich Kunststoffverpackungen, die Kunststoffnichtverpackungen werden über die Restmüllsammlung entsorgt. Dieser Anteil an Kunststoffen entgeht somit dem Recycling. Rein gewerbliche Kunststoffverpackungen werden separat gesammelt. Andere Kunststoffabfälle (Nichtverpackungen) werden mit dem Rest- oder Sperrmüll erfasst. In Recyclingzentren werden teilweise Kunststoffnichtverpackungen getrennt gesammelt, um sie entweder stofflich wiederzuverwerten oder als Ersatzbrennstoff für die Zementindustrie zur Verfügung zu stellen. Für die Berechnung der Kunststoffrecyclingrate wird die aus den Leichtverpackungssortierungsanlagen aussortierte Ver- packungsmenge (inkl. eines kleinen Anteils von Mischkunststoffen), den gesamten in der Abfallwirtschaft vorhanden Kunststoffverpackungen (im gemischten Siedlungsabfall, Gewerbemüll, Sperrmüll, getrennter Sammlung) gegenübergestellt. Im Gegensatz zu anderen Ländern, wo ein $100-\%$-Recycling bereits bei den angelieferten Mengen anerkannt wird (also eine Input-bezogene Berechnung), wird in Österreich bereits eine Output-bezogene Berechnung durchgeführt. Trotz der vergleichsweise realitätsnahen Berechnungsmethode werden aber immer noch nicht die Mengen der in Verkehr gebrachten Kunststoffnichtverpackungen in die Recyclingquote einbezogen. Aktuell erreicht Österreich laut Plastics (2015b) eine Recyclingrate von 26 M-\% (Abb. 1).

Wird zum Beispiel der EU-Schnitt von 29,7 M-\% Recyclingrate genauer betrachtet (siehe Abb. 1), berechnet stoffmengen und nicht vom gesamten Kunststoffaufkommen. Unter Berücksichtigung des Jahreskunststoffbedarfes würde sich die Recyclingrate deutlich verringern. Ein derartiges Bild ist auch in Japan erkennbar. In Australien wird bei einer ähnlichen Recyclingrate vergleichsweise mehr gesammelt. Die Sammelmengen in den Vereinigebenso höher als in den anderen Vergleichsstaaten, gleichwohl sich eine relativ niedrige Recyclingrate zeigt. Das kann darauf deuten, dass die Kunstsich diese aus den gesammelten Kunstten Staaten von Amerika (USA) sind stoffe zuvor gesammelt werden, jedoch anschließend nicht recycelt, sondern deponiert oder thermisch verwertet werden.

Die inputseitige Berechnungsmethode spiegelt sich auch in den Massenbilanzen der Leichtverpackungssortieranlagen wieder. Der überwiegende Stoffstrom, Mischkunststoffe $(50$ bis $60 \mathrm{M}-\%$ vom Input), der die Anlage nach der Abtrennung der sortenreinen Kunststoffe verlässt, besitzt immer noch ein hohes Recyclingpotenzial (Polyolefingehalt von $>40$ M-\% (Kranzinger et al. 2016)). Wenngleich eine zukunftsorientierte Abfallwirtschaft unter Berücksichtigung der Abfallhierarchie lt. EU-Abfallrichtlinie 2008/98/EG das Recycling über andere Verwertungsmöglichkeiten (z. B. thermische Verwertung) stellt, wird dieser Stoffstrom derzeit für die Herstellung von Ersatzbrennstoffen eingesetzt. In anderen Worten, dem stofflichen oder rohstofflichen Kunststoffrecycling entgehen rund $60.000 \mathrm{t}$ an recyclingfähigen Kunststoffen pro Jahr. Das ist ungefähr die gleiche Menge, die tatsächlich von den österreichischen Leichtverpackungssortieranlagen zum stofflichen Recycling übergeht.

Parallel dazu werden aus den gemischten Siedlungsabfällen weniger als $5 \mathrm{M}-\%$ an Wertstoffen (davon hauptsächlich Metalle) aussortiert und recycelt. Die restlichen $95 \mathrm{M}-\%$ werden direkt thermisch bzw. indirekt über Mechanische (MA) oder MechanischBiologische Abfallaufbereitungsanlagen (MBA) aufbereitet und energetisch verwertet. Folglich kommt ein Großteil der noch im Restmüll befindlichen und wiederverwertbaren Kunststoffe dem Recycling abhanden. Kranzinger et al. (2016) berichten von einem Polyolefinpotenzial von über $110.000 \mathrm{t} / \mathrm{a}$ (Tonnen pro Jahr) in den gemischten Siedlungsabfällen. Diese Menge zeigt, dass das Polyolefinpotenzial im Restmüll beinahe gleich groß ist wie jenes in der getrennten Sammlung. Lediglich mit dem Unterschied, dass die Kunststoffe im Restmüll in der Praxis nicht recycelt werden.

Ungeachtet des großen noch ungenutzten Rohstoffpotenzials gibt es momentan wenig Anreize, die recyclingfähigen Kunststoffe (hauptsächlich Polyolefin-Verpackungen) verstärkt von der thermischen Verwertung in das Recycling umzulenken. In naher $\mathrm{Zu}$ kunft könnte aber der am 02.12.2015 veröffentlichte Vorschlag der Europäischen Kommission zum Kreislaufwirt- 


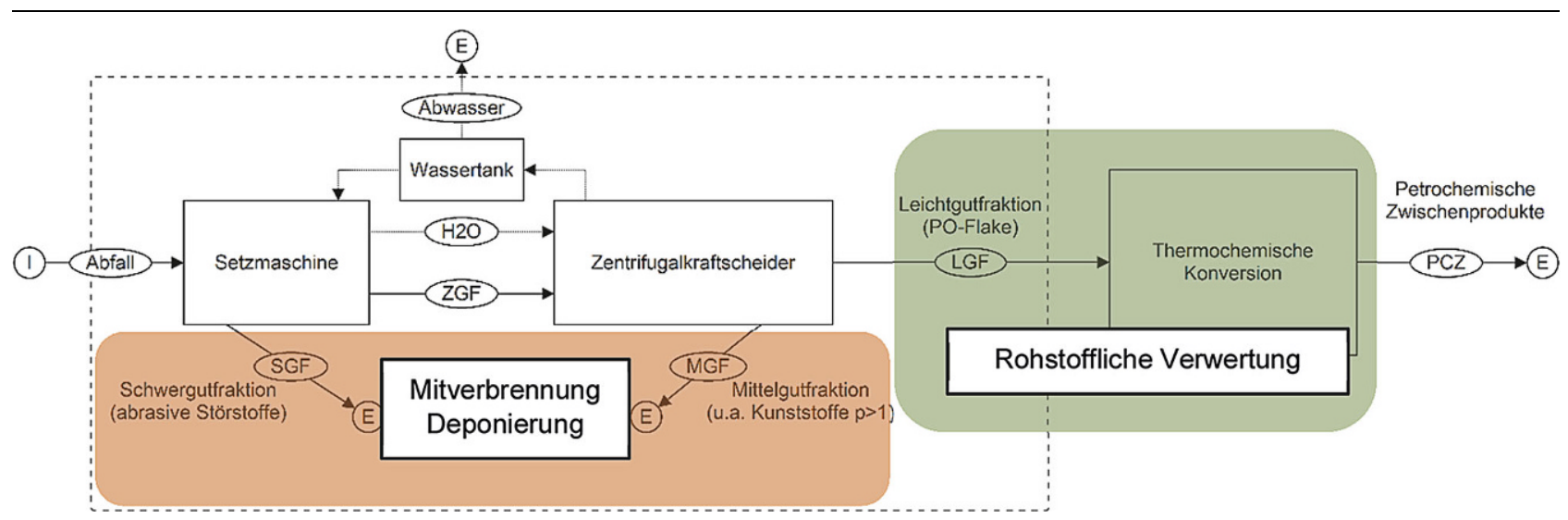

Abb. 2 Vereinfachtes Prozessschema der nass-mechanischen Aufbereitungsanlage mit den Verwertungswegen der Trennfraktionen sowie dem Abwasser. (SGF Schwergutfraktion, ZGF Zwischengutfraktion, MGF Mittelgutfraktion, LGF Leichtgutfraktion, H2O Prozesswasser, PCZ Petrochemische Zwischenprodukte, E Prozessende, I Abfallinput)

schaftspaket neue Dynamik in die derzeit noch ausbaufähige österreichische Kunststoffrecyclinglösung bringen. Die Richtlinie sieht eine neue europaweit gültige Vorgabe zur Erhöhung der Recyclingziele vor. Für Siedlungsabfälle ist eine vorläufige Recyclingquote von $65 \mathrm{M}-\%$ bis zum Jahr 2030 festgelegt (EK 2015c), wobei momentan Verhandlungen über eine weitere Erhöhung laufen (EK 2017b). Laut BMLFUW (2015) erfüllt Österreich beinahe jetzt schon diese Zielvorgabe. Für Verpackungsabfälle aus Kunststoff wird eine Recyclingquote von $55 \mathrm{M}-\%$ bis zum Jahr 2030 angestrebt (EK 2015b). Auch bei dieser Richtlinie gibt es momentan Verhandlungen über die endgültige Höhe der Quote (EK 2017a). Aufgrund einer möglichen Änderung der Berechnungsmethode der Quoten (Bezugsgröße wird von den Inputmengen auf die Outputmengen der Sortieranlagen geändert) könnte es aber passieren, dass die aktuelle Recyclingquote für Siedlungsabfälle fällt und ausgeglichen werden muss. Sollte sich das bewahrheiten, würde Österreich zusätzliche Recyclingmengen benötigen. Einen Beitrag zur Erhöhung der Recyclingquoten könnten speziell die hochwertigen und recyclingfähigen Polyolefine liefern. Diese umfassen den Großteil der in der Restmüll- und den in der getrennten Verpackungssammlung befindlichen Kunststoffe.

\subsection{Projekthintergrund und -zie}

Während konventionelle trocken-mechanische Abfallaufbereitungsanlagen standardmäßig Abfälle zu hoch- und mittelkalorischen Ersatzbrennstoffen umsetzten, zielt das Research Studio
Austria „Plastic Reborn“ auf die neuartige nass-mechanische Aufbereitung von kunststoffreichen Abfallströmen zur Anreicherung von Polyolefinfraktionen. Im Forschungsprojekt arbeiten drei Lehrstühle der Montanuniversität Leoben, der Lehrstuhl für Aufbereitung und Veredlung, der Lehrstuhl für Verfahrenstechnik des industriellen Umweltschutzes und der Lehrstuhl für Abfallverwertungstechnik und Abfallwirtschaft sowie industrielle Partner zusammen. Das Konsortium befasst sich mit der Fragestellung, ob die Abtrennung hochreiner Polyolefinfraktionen als Vorbereitung für ein rohstoffliches Recycling mittels thermochemischer Konversion technisch und wirtschaftlich umsetzbar ist.

\section{Material \& Methode}

\subsection{Identifikation polyolefinreicher Abfallströme}

Unter Verwendung des Abfallkatalogs nach ÖNORM S 2100 (AS 2005) und einer anschließenden Literaturrecherche wurden aus über 1500 Abfallschlüsselnummern jene polyolefinreichen Abfallströme ausgewählt, die anhand von vordefinierten Ausschlusskriterien (siehe Kranzinger et al. 2016) für die nassmechanische Aufbereitung einsatzfähig sind. Sieben potenzielle Abfallströme aus den Bereichen Leichtverpackungssortierung, Ersatzbrennstoffe, Rejects (Spuckstoffe) und dem Landfill Mining konnten ausfindig gemacht werden. Ein genauer Polyolefingehalt der Abfallströme konnte alleine mit einer Literaturrecherche nicht ermittelt werden. Infolgedessen wurden Probemateriali- en organisiert und mittels Dichtetrennung sowie mittels Fourier-TransformInfrarotspektrometer untersucht. Parallel dazu wurde der Ist-Stand der österreichischen Abfallwirtschaft modelliert (Verwendung der STAN Software [Cencic \& Rechberger 2008]). Relevante abfallwirtschaftliche Aufbereitungsanlagen, die für die Behandlung der polyolefinreichen Abfallströme in Betracht kommen, wurden in einem Abfallmengenflussbild dargestellt. Somit konnten die wichtigsten Polyolefinströme und deren Wege in der österreichischen Abfallwirtschaft abgebildet werden.

\subsection{Anlagenkonzept und Verwertungswege der Trennfraktionen}

Die Versuchsanlage im Technikumsmaßstab kann prinzipiell als eine Erweiterung der überwiegend trockenabfallwirtschaftlichen Infrastruktur angesehen werden. Neben dem positiv zu bewertenden Reinigungseffekt, hebt sich die nass-mechanische Dichtesortierung durch eine erhöhte Selektivität und die daraus resultierende Trenngüte (-effizienz) von den Trockentrennverfahren ab.

Der in Abb. 2 beschriebene Prozessaufbau dient zur Gewinnung einer polyolefinreichen Altkunststofffraktion, dem PO-Flake bzw. der Leichtgutfraktion (LGF). Diese LGF soll am Prozessende mittels einer thermo-chemischen Konversion in petrochemische Intermediates überführt werden, welche in einer konventionellen Raffinerie im besten Fall zu Grundprodukten (Ethen, Propylen) für die neuerliche Kunst- 
stoffsynthese (rohstoffliches Recycling) weiterverarbeitet werden.

In einem ersten Schritt wurden die sieben Abfallfraktionen mittels Einwellenzerkleinerer auf eine Korngröße von $<20 \mathrm{~mm}$ (Millimeter) zerkleinert, um etwaigen Verstopfungen im Bereich des Zentrifugalkraftscheiders vorzubeugen. Nach der Zerkleinerung wurde das Probenmaterial über einen Aufgabebehälter in die Setzmaschine aufgegeben. In der Setzmaschine erfolgt durch Absinken der spezifisch schwereren Materialien die erste Trennung. Durch induzierte Hubfrequenzen bildet sich ein sogenanntes Setzbett aus, in dem sich dichtere Störstoffe in der unteren Schicht und die weniger dichten Kunststoffe in der oberen Schicht anordnen. Am Austrag der Setzmaschine wird das Setzbett mittels Leitblechen in die Schwergutfraktion (SGF) und in die Zwischengutfraktion (ZGF) geteilt. Die SGF wurde aus dem Prozess abgetrennt und die ZGF (polyolefinreich) über eine Dosiereinrichtung in den Zentrifugalkraftscheider weitergeleitet.

Im Zentrifugalkraftscheider werden aufgrund des Dichteunterschieds des Trennmediums (Wasser) und der Abfallmaterialien spezifisch leichtere Materialien (PO-Flake bzw. LGF) infolge des Auftriebs von den spezifisch schwereren Materialien (MGF, andere Kunststoffe und Störstoffe) separiert. Zur Beschleunigung der Trennung wird dieses Prinzip hier in das sich ausbildende stärkere Zentrifugalkraftfeld überführt und ausgenutzt. Dabei reichert sich das Leichtgut an der Grenzfläche zwischen Wasser- und Luftwirbel an und wird durch die Schwerkraft über die untere axiale Öffnung ausgetragen. Jene Partikel mit einer Dichte $>1 \mathrm{~g} / \mathrm{cm}^{3}$ (Gramm pro Kubikzentimeter) werden von der Zentrifugalkraft an den Zylindermantel gedrückt und mit dem Wasser als Mittelgutfraktion ausgetragen. Alle drei Fraktionen (LGF, MGF \& SGF) werden am Ende über ein Linearschwingsieb entwässert. Der Trockensubstanzgehalt spielt vor allem für die Nutzung des MGF als Ersatzbrennstoff in der Zementindustrie eine wichtige Rolle. Die nass-mechanische Aufbereitung und die rohstoffliche Verwertung der LGF macht nur Sinn, wenn auch die nichtpolyolefinhaltigen Outputfraktionen (SGF und MGF) ökonomisch sinnvoll verwertbar sind.

\subsection{Analytisches Monitoring}

Für die Verwertung der MGF und SGF ist die Mitverbrennung in Anlagen gemäß $\S 3$ (6) Abfallverbrennungsverordnung (BMLFUW 2010) angedacht. Die beiden Outputfraktionen wurden auf die Einhaltung der gesetzlichen Grenzwerte und das Prozesswasser wurde auf potenzielle Grenzwertüberschreitungen nach Anlage A $\$ 4$ der Abwasseremissionsverordnung (AAEV) (BMLFUW 1996) analysiert. Die Ergebnisse lassen abschätzen, inwieweit Abwassernachbehandlungsanlagen (mechanische oder chemisch/biologische Reinigung) zur Begrenzung der Frachten und Konzentrationen schädlicher Inhaltsstoffe nötig sind, um gesetzlich zulässig in die Kanalisation einzuleiten zu können.

\section{Ergebnisse und Interpretation}

\subsection{Polyolefinpotenzial in der} österreichischen Abfallwirtschaft

Das Abfallmengenflussbild in Abb. 3 projiziert den derzeitigen logistischen und quantitativen Zusammenhang zwischen Sammlung, Sortierung und Verwertung der sieben polyolefinreichen Abfallströme in der österreichischen Abfallwirtschaft. Die Abfallströme sind in drei Entstehungssektoren (Haushalt, Gewerbe und Industrie) zusammengefasst, wobei die Leichtverpackungsabfälle (LVP) aus der getrennten Sammlung und der gemischte Siedlungsabfall mit einer Jahresmenge von 1,6 Mio. $t$ das größte Abfallpotenzial für die nassmechanische Aufbereitung bilden (bei den LVP sind die gewerblichen Mengen inkludiert). Gewerbeabfälle sind die zweitgrößten Abfallströme mit einer Jahrestonnage von 0,6 Mio. t. Abfälle aus der Industrie sind mit beinahe 0,2 Mio. $t$ das kleinste Mengenpotenzial (jedoch ist deren Polyolefinanteil am höchsten (Kranzinger et al. 2016)). Insgesamt sind das 2.456.200 t an potenziellen polyolefinreichen Abfallströmen.

Im Detail beinhaltet das Abfallmengenflussbild das werkstoffliche Recycling, die Sortierung in einer LVP-Anlage, die direkte Verbrennung von unbehandeltem gemischtem Siedlungsabfall, Gewerbeabfall und Produktionsabfall in Müllverbrennungsanlagen (MVA) sowie die Aufbereitung in einer MBA oder MA mit Ersatzbrennstoff (EBS)Aufbereitung. Das Flussbild umfasst auch die Mitverbrennung von EBS in ei- nem Zementwerk und die Verbrennung der Rückstände aus der mechanischen Aufbereitung in einer Wirbelschichtanlage.

Ein großer Teil der Abfälle aus Gewerbe und Haushalten (gemischter Siedlungsabfall mit $1.026 .700 \mathrm{t}$ ) wird ohne Vorbehandlung in thermischen Behandlungsanlagen für Siedlungsabfall (MVA mit Rostfeuerung) eingesetzt. Weitere 241.700 t gelangen in MBA und die restlichen $163.200 \mathrm{t}$ werden in einer MA behandelt. Die Leichtverpackungen aus dem Gelben Sack und der Gelben Tonne $(225.000 \mathrm{t})$ gelangen in die LVPSortierung. Etwa $10 \%$ des Inputs werden dort über den Siebdurchgang in die MA weitergeleitet und ein Drittel verlässt die Anlage als sortenreiner Kunststoff. Den größten Strom, der die LVPSortieranlage verlässt, stellt die Mischkunststofffraktion dar. Diese Fraktion umfasst $123.500 \mathrm{t}$ und wird $\mathrm{zu}$ EBSAufbereitungsanlagen transportiert. In diesen Anlagen werden EBS für die Mitverbrennung in Zementwerken erzeugt. Für das werkstoffliche Recycling sind $79.000 \mathrm{t}$ sortenreine Kunststoffe im System vorhanden. Während der Vorbereitung für das werkstoffliche Recycling werden mittels Sink-SchwimmTrennung noch $4000 \mathrm{t}$ sortenfremde Kunststoffe abgetrennt, die das System verlassen. Die restlichen Kunststoffe werden zerkleinert, extrudiert und regranuliert.

Die 241.700t an Abfall, die in die MBA gelangen, teilen sich auf in $107.600 \mathrm{t}$ heizwertreiche Fraktion, 91.300 t Deponiematerial, $4100 \mathrm{t}$ Recyclingmaterial (hauptsächlich Metalle) und in $38.700 \mathrm{t}$ Rotteverlust. Während die heizwertreiche Fraktion der Mitverbrennung zugeführt wird, verlassen die drei anderen Ströme das System.

$\mathrm{Zu}$ den 163.200t an Gewerbe-, gemischten Siedlungs- und Produktionsabfällen, welche in der MA behandelt werden, kommen noch $22.500 \mathrm{t}$ Siebdurchgang aus der LVP-Sortierung hinzu. Die Summe dieser Abfälle teilt sich auf in $61.700 t$ Recyclingmaterial und Verluste, die das System verlassen, 311.800 t EBS für die Mitverbrennung und $311.800 \mathrm{t}$ heizwertreicher Fraktion für die Abfallverbrennung in einer Wirbelschichtanlage. Von den $311.800 t$ EBS gehen $192.800 \mathrm{t}$ zur Mitverbrennung ins Zementwerk. Die restlichen 119.000t EBS werden ins Ausland exportiert. Diese Exporte ergeben sich aus der EBS-Nachfrageobergrenze der österreichischen Zementwerke. 


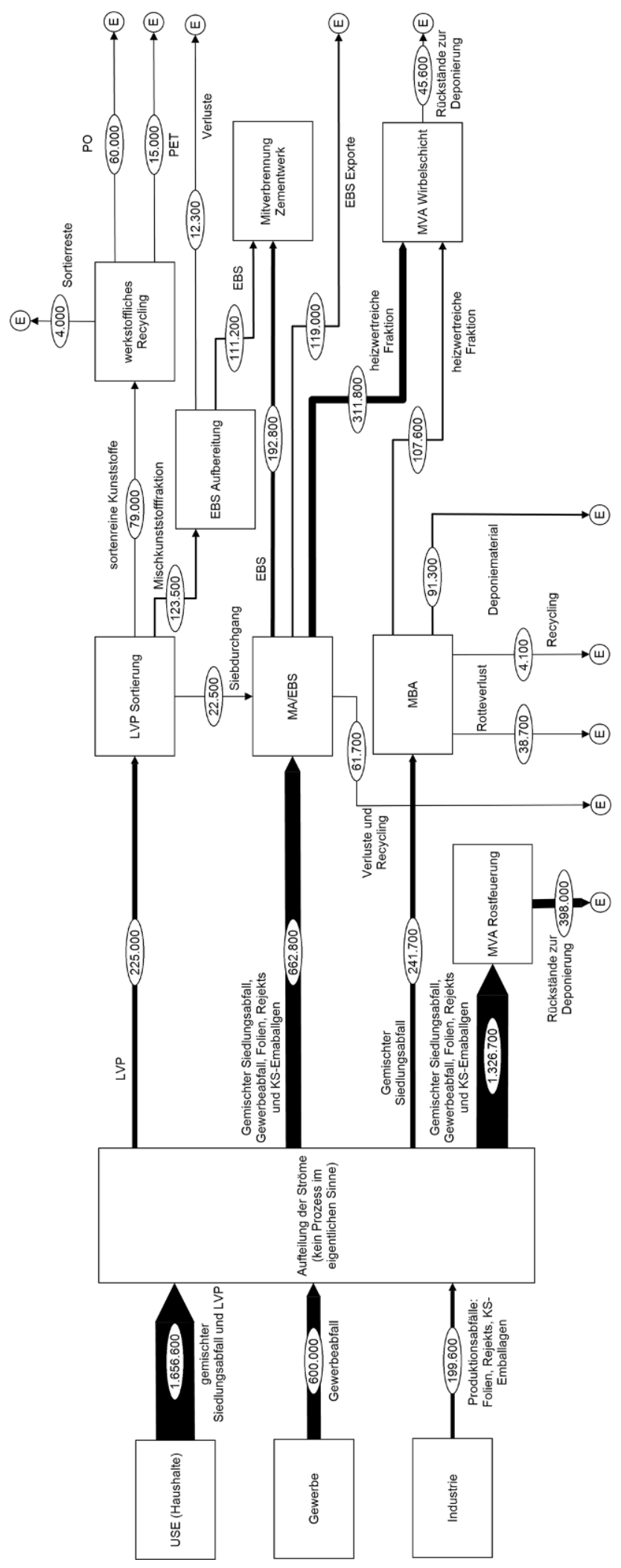

Abb. 4 zeigt die Mengenverteilung auf der Ebene der Polyolefine (Stoffebene). Etwa 60.000t an Polyolefinen gelangen pro Jahr aus der LVP-Sortierung ins werkstoffliche Recycling. Die restlichen $15.000 \mathrm{t}$ sortenreine Kunststoffe bestehen hauptsächlich aus PET. Im Siebdurchgang, der aus der LVPSortierung zur MA/EBS-Anlage gelangt, befinden sich $30 \%$ Polyolefine. Die restlichen Polyolefine aus den LVP-Sortieranlagen werden mit der Mischkunststofffraktion in eine EBS-Aufbereitungsanlage verschoben. Weitere $161.800 \mathrm{t}$ an Polyolefinen werden über MA/EBS-Anlagen zu EBS, einer heizwertreichen Faktion und als EBS exportiert. Ein kleinerer Anteil (19.100 t) wird bei den MBA aufbereitet und in Mitverbrennungsanlagen thermisch verwertet. Die übrigen $123.100 t$ Polyolefine fließen in die direkte Abfallverbrennung.

\subsection{Schadstoffbelastung des Prozessabwassers}

Erste Ergebnisse zeigen ein erhöhtes $\mathrm{BSB}_{5}$ :CSB-Verhältnis (Biologischer und Chemischer Sauerstoffbedarf) bei allen Abfallströmen. Einzelne Abwasserproben erreichen ein $\mathrm{BSB}_{5}$ :CSB-Verhältnis von bis zu 1:26. Ein Verhältnis von bis zu 2 deutet auf ein biologisch gut abbaubares Abwasser hin. Je größer das Verhältnis wird, desto schlechter wird die Abbaubarkeit (N.N. 2000). Bei einigen Abfallströmen ist auch der Grenzwert der absetzbaren Stoffe überschritten. Außerdem weist ein Abfallstrom eine erhöhte Konzentration von organischen Halogenverbindungen $(0,7 \mathrm{mg} / \mathrm{L}$ (Milligramm pro Liter) AOX) auf. Der Grenzwert lt. AAEV liegt für AOX bei $0,5 \mathrm{mg} / \mathrm{L}$.

Möglichkeiten, um dem erhöhten CSB und den AOX-Konzentrationen entgegenzuwirken, könnten nachgeschaltete physikalisch-chemische Abwasserbehandlungsmethoden (Adsorption, Flotation, Ionenaustauscher, Nassoxidation, Filtration etc.) sein. Zusätzlich würde ein Sedimentationsbecken die Konzentration der absetzbaren Stoffe verringern.

\subsection{Schadstoffkonzentrationen It. Abfallverbrennungsverordnung}

Im Gegensatz zur direkten thermischen Verwertung sind für die Mitverbrennung inputseitige Grenzwerte für die Abfälle gesetzlich vorgeschrieben und müssen eingehalten werden. Diese sind 

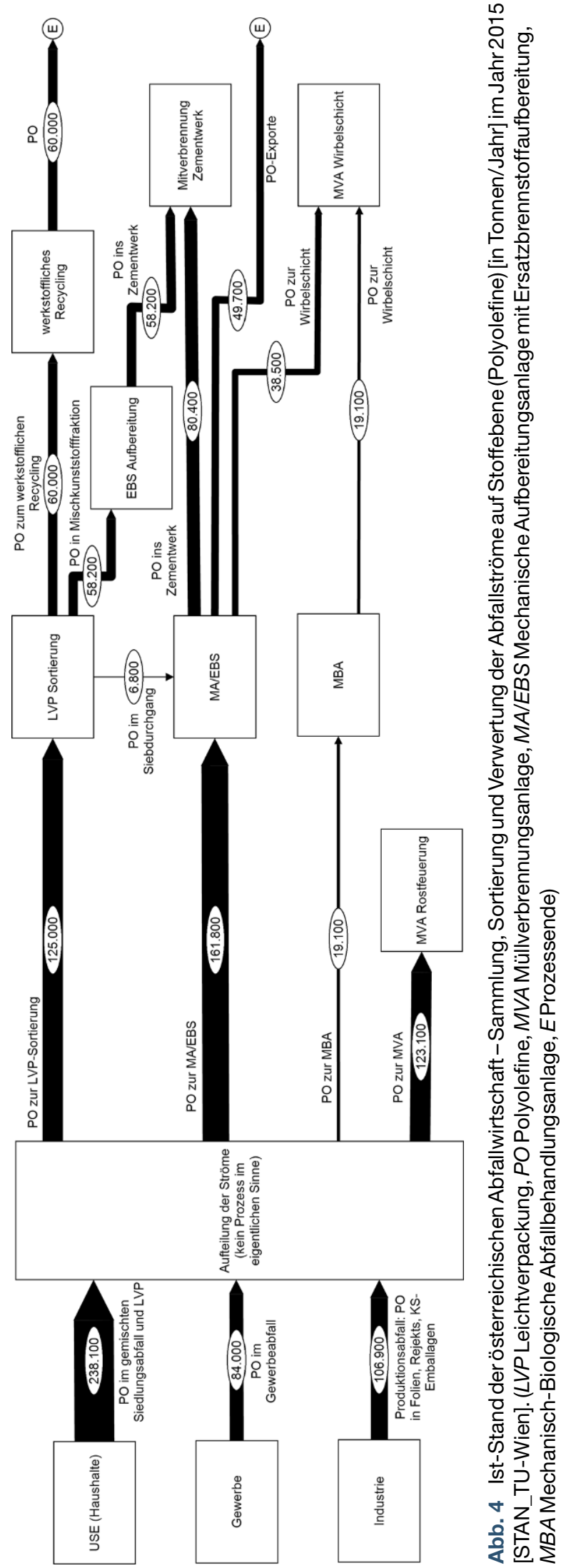

in Anlage 8 der Abfallverbrennungsverordnung aufgelistet (BMLFUW 2010).

Die Grenzwerte von Arsen, Chrom $_{\text {gesamt }}$ und Quecksilber zeigen in Abb. 5 bei allen Abfallströmen (A1 bis A7) keinerlei Überschreitung. Für den Grenzwert Antimon sind jedoch bei Abfallstrom A2 und A5 Überschreitungen in der Schwergutfraktion auffällig. Außerdem sind die Schadstoffkonzentration in der Mittelgutfraktion von A3 und die Schwergutfraktion A6 und A7 knapp unter dem gesetzlichen Grenzwert. Die erhöhte Schadstoffkonzentration bzw. die Grenzwertüberschreitung kann auf einen hohen Polyvinylchlorid (PVC)- und PET-Gehalt zurückzuführen sein, weil Antimon als Stabilisator in der PVC-Herstellung zum Einsatz kommt und bei der PET-Herstellung als Katalysator wirkt. Zusätzlich kann das Bindemittel in der Druckfarbe bei A2 für die erhöhte Schadstoffkonzentration verantwortlich sein.

Das Schwermetall Blei und die Grenzwertüberschreitung bei A2, A3, A5 und A6 können auf den PVC-Anteil zurückzuführen sein. Das Schwermetall kommt, wie auch Antimon, bei der Herstellung des Kunststoffs als Stabilisator zum Einsatz. Bei A2 dürfte die Überschreitung eher auf das Bindemittel in der Druckfarbe zurückzuführen sein, als auf die PVC-Partikel selbst.

Erhöhte Cadmiumkonzentrationen der Abfallströme A5 und A7 können auf die vermehrte Verwendung des Schwermetalls als Stabilisierungsmittel bei PVC zurückzuführen sein. Wiederum kann der Anteil von Druckerfarbe den hohen Cadmiumwert von A2 erklären. Der beträchtliche Anteil von Cadmium in A4 kann durch den Polystyrolanteil bedingt sein, da dieser Stoff aufschwimmt und von der Setzmaschine nicht in die SGF abgetrennt werden kann.

\subsection{Verwertungspotenzial der Outputfraktionen}

Für die thermische Verwertung der SGF und MGF in Mitverbrennungsanlagen als EBS müssen einerseits die gesetzlichen Grenzwerte der Schadstoffkonzentrationen und andererseits die von der Mitverbrennungsanlage geforderten Spezifikationen (Aschegehalt, Schwefelgehalt, Heizwert, Chlorgehalt, Korngröße etc.) eingehalten werden. Eine der wichtigsten Spezifikationen für die Anlagenbetreiber ist der ausreichende Energiegehalt bzw. Heizwert des Ersatzbrennstoffs. Zum Beispiel 


\section{Originalarbeit}

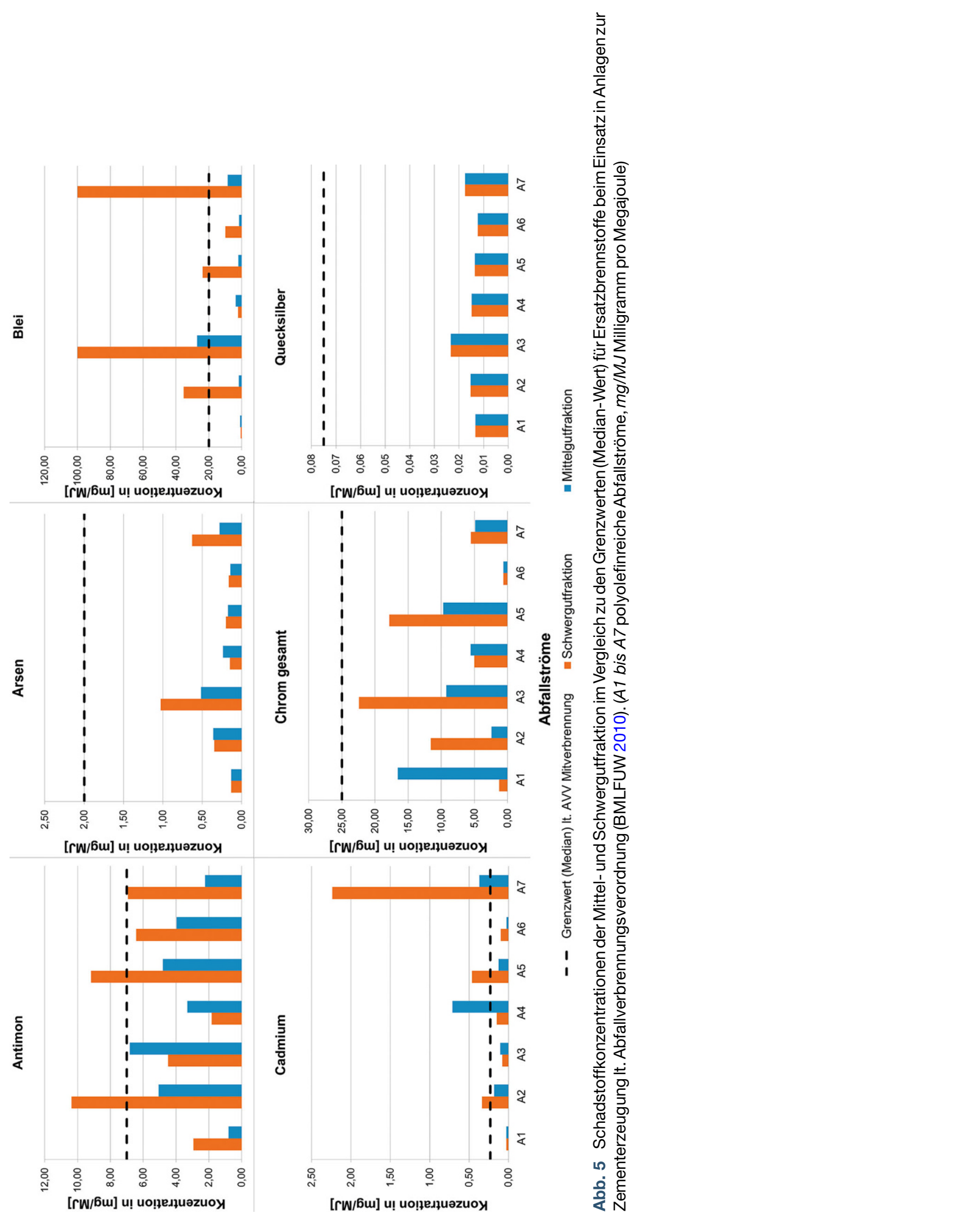




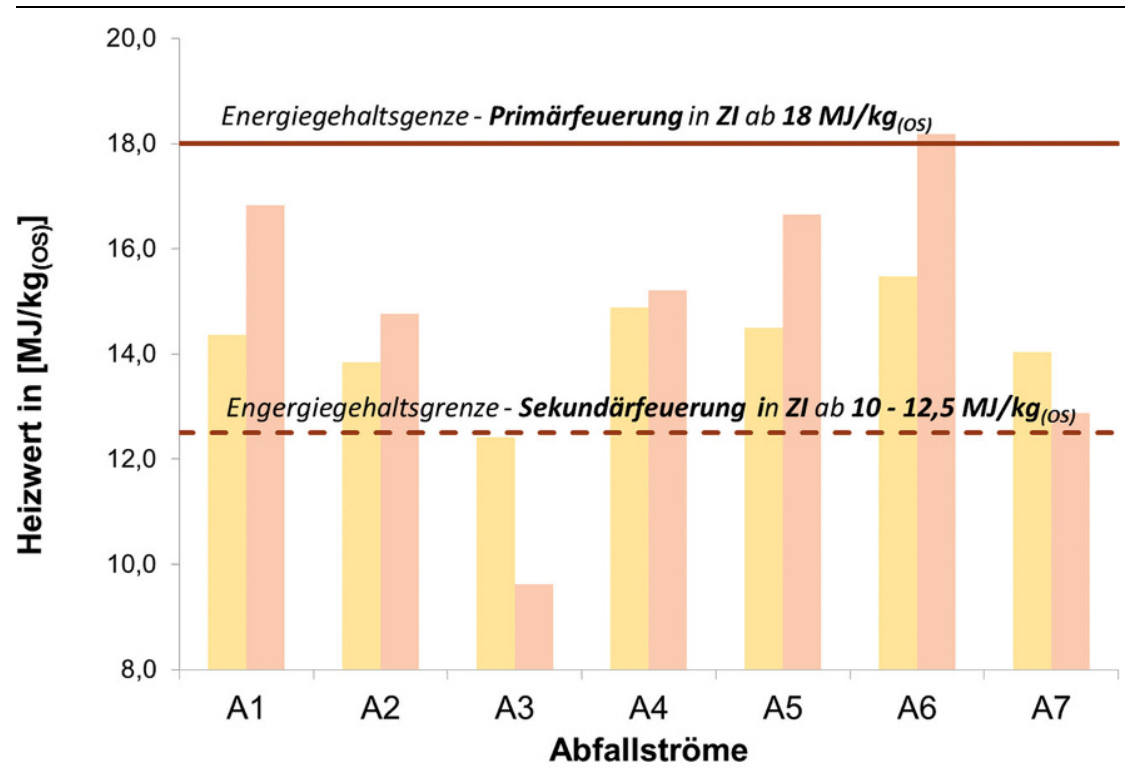

Mittelfratkion $\quad$ Schwerfraktion

Abb. 6 Thermisches Verwertungspotenzial der Abfallströme nach Entwässerung, bezogen auf den jeweiligen Energiegehalt der Schwergutfraktion bzw. der Mittelgutfraktion. (ZI Zementindustrie, $H u_{(O S)}$ Heizwert der Originalsubstanz, $A 1$ bis $A 7$ polyolefinreiche Abfallströme, $M J / k_{(O S)}$ Megajoule pro Kilogramm von der Originalsubstanz)

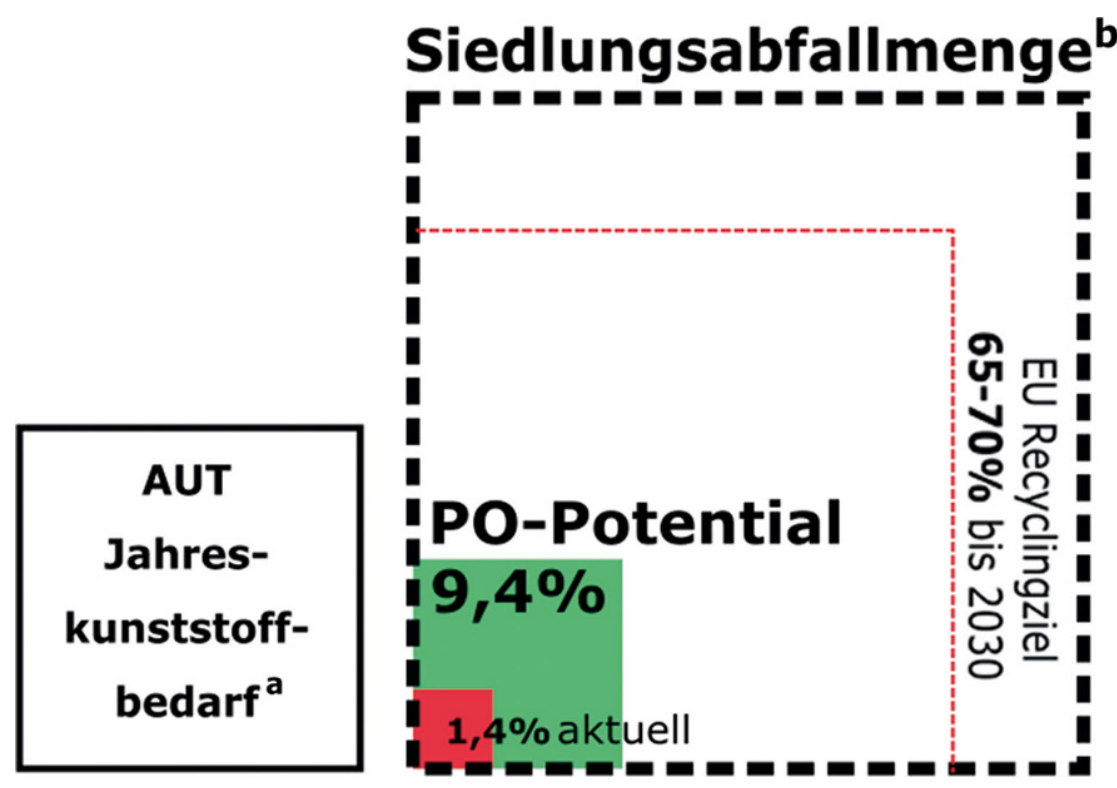

Abb. 7 Theoretischer Beitrag der Polyolefinabfälle zum EU-Recyclingziel 2030 Siedlungsabfälle aus Haushalten und ähnlichen Einrichtungen in Österreich. a Zusammensetzung: 0,5 Mio. t Polyolefine und 0,6 Mio. t andere Kunststoffe (Plastics 2015a); b 4,2 Mio. t Siedlungsabfälle aus Haushalten und ähnlichen Einrichtungen im Jahr 2014 (BMLFUW 2015) wird für die Mitverbrennung in der Sekundärfeuerung (Brennstoff für die Calzinatorfeuerung oder für den Drehrohreinlauf) eines Zementwerks ein Heizwert von 10 bis $12 \mathrm{MJ} / \mathrm{kg}_{(\mathrm{OS})}$ (Megajoule pro Kilogramm von der Originalsubstanz) benötigt. In der Primärfeuerung werden noch höhere Energiegehalte ab $18 \mathrm{MJ} / \mathrm{kg}_{(\mathrm{OS})}$ gefordert (Deditz et al. 2014; Sarc et al. 2014; Sarc \& Lorber 2013).

Die Versuche im Technikum zeigen, dass nur wenige SGF direkt nach der Entwässerung am Linearschwingsieb als Ersatzbrennstoff für die Sekundärfeuerung zum Einsatz kommen können, da der Wassergehalt zu hoch ist. Die restlichen SGF sowie MGF wären jedoch für die Mitverbrennung in einer Wirbelschichtverbrennung einsetzbar. Da die Zuzahlungen für die thermische Verwertung als Ersatzbrennstoff in der Sekundärfeuerung niedriger sind als für die Wirbelschichtverbrennung, sollten die Outputfraktionen aus wirtschaftlicher Sicht weiter konditioniert werden. Eine nachgeschaltete Entwässerung erscheint dafür sinnvoll. Ausgehend von Entwässerungsversuchen kann ein realistischer Minimalwassergehalt von $10 \mathrm{M}-\%$ für die SGF und $35 \mathrm{M}$-\% für die MGF angenommen werden. Die Ergebnisse in Abb. 6 zeigen, dass durch eine zusätzliche Entwässerung der Heizwert bei fast allen Abfallströmen um mehr als $3 \mathrm{MJ} / \mathrm{kg}_{(\mathrm{OS})}$ ansteigt. Auffällig ist die extreme Energiezunahme bei A2 und A3. Das kann auf den hohen Celluloseund Textilanteil zurückgeführt werden. Dieses Material ist stark wasseraufnahmefähig, was wiederum $\mathrm{zu}$ einem durchschnittlich höheren Wassergehalt führt.

Insgesamt kann davon ausgegangen werden, dass die SGF und MGF nach einer zusätzlichen Entwässerung als Ersatzbrennstoff in der Zementindustrie zum Einsatz kommen können. Allerdings müssen noch weitere Spezifikationen (Chlorgehalt, Korngröße etc.) ausgewertet werden. Ein besser entwässerter Abfallstrom verringert außerdem die Transportmengen und spart somit Kosten und Kohlendioxidemissionen (in Form von eingespartem Treibstoff).

\section{Schlussfolgerungen}

Grundsätzlich zeigt die Erhebung des Polyolefinpotenzials, dass trotz einer gut funktionierenden Abfalltrennung immer noch ein großes ungenutztes Kunststoffrecyclingpotenzial in der ös- 
terreichischen Abfallwirtschaft vorhanden ist. Speziell die hochwertigen und recyclingfähigen Polyolefine können mit einem theoretischen Jahrespotenzial von $429.000 t$ beziffert werden. Diese werden derzeit in den trocken-mechanischen und mechanisch-biologischen Abfallbehandlungsanlagen überwiegend $\mathrm{zu}$ hochkalorischen Ersatzbrennstoffen aufbereitet (86 M-\%) und entgehen somit dem Recycling. Anhand dieses Abfallmengenflussbildes können außerdem logistische und standortspezifische Überlegungen für die Errichtung einer solchen nass-mechanischen Aufbereitungsanlage angestellt werden.

Die Trennversuche mittels des nassmechanischen Aufbereitungsverfahrens zeigen, dass über $90 \mathrm{M}-\%$ der in den Abfallströmen enthaltenen Polyolefine als polyolefinreiches Vorkonzentrat in die Leichtgutfraktion übergeführt wurden (Bauer et al. 2017). Diese Mehrmengen an recyclingfähigen Kunststoffen (gewonnen aus den Abfallströmen Leichtverpackungssammlung, gemischte Siedlungsabfälle inkl. Gewerbeabfälle und Produktionsabfälle) könnten einen positiven Beitrag zur Erfüllung der vorgeschlagenen Recyclingziele der Europäischen
Kommission haben. Seit 2014 werden im Richtlinienpaket zur Kreislaufwirtschaft („Towards a circular economy: a zero waste programme for Europe") (EK 2015a) für Siedlungsabfälle und für Kunststoffverpackungen Recyclingquoten verhandelt (siehe Einleitung). Anhand der gegenwärtigen Neuentwürfe (EK 2017a, 2017b) ist zu erwarten dass die erwähnten Recyclingquoten noch etwas höher ausfallen werden und somit weitere Stoffströme aus der Deponierung und der Verbrennung in das Recycling umgelenkt werden müssen, um die höheren Recyclingziele zu erreichen.

Theoretisch haben die rückgewonnenen Mengen aus „Plastic Reborn“ und die anschließende rohstoffliche Verwertung ein Recyclingpotenzial von $9,4 \mathrm{M}-\%$, bezogen auf das jährliche Siedlungsabfallaufkommen aus Haushalten und ähnlichen Einrichtungen (Abb. 7, die Größe der Flächen beschreibt proportional das jährliche Abfallaufkommen bzw. den Bedarf). Verglichen mit dem aktuellen Beitrag der Kunststoffrecyclingbranche (1,4 M-\%) würde das eine Erhöhung um $8 \mathrm{M}-\%$ ausmachen Die Berechnung basiert auf den Outputmengen der Sortieranlagen.
Die Trennversuche zeigen außerdem, dass die Restfraktionen (Mittelgutfraktion und Schwergutfraktion) sehr wahrscheinlich in Wirbelschichtöfen und Drehrohröfen als Ersatzbrennstoff verwertet werden können. Diese zur energetischen Verwertung vorgesehenen Restfraktionen zeigen keine Überschreitung der gesetzlich vorgeschriebenen Inputgrenzwerte für die Mitverbrennungsanlagen.

Acknowledgements Open access funding provided by Montanuniversität Leoben.

Open Access Dieser Artikel wird unter der Creative Commons Namensnennung 4.0 International Lizenz (http:// creativecommons.org/licenses/by/4. 0/deed.de) veröffentlicht, welche die Nutzung, Vervielfältigung, Bearbeitung, Verbreitung und Wiedergabe in jeglichem Medium und Format erlaubt, sofern Sie den/die ursprünglichen $\mathrm{Au}$ tor(en) und die Quelle ordnungsgemäß nennen, einen Link zur Creative Commons Lizenz beifügen und angeben, $o b$ Änderungen vorgenommen wurden.
AS (Austrian Standards Institut) (2005): ÖNORM S2100 Abfallkatalog. Wien, Österreich A'Vard, D. \& Allan, P. (2014): 2013-14 National Plastics Recycling Survey, Final Report. http://www.packagingcovenant.org.au/data/ Publications/R03-03-A11013_NPRS_2013-14_Re port.pdf. Gesehen 7. März 2017

Bauer, M., Lehner, M., Schwabl, D., Flachberger, H., Kranzinger, L., Pomberger, R. \& Hofer, W. (2017): Bestandsaufnahme und mögliche Perspektiven der nass-mechanischen Aufbereitung von Altkunststoffen für das rohstoffliche Recycling. Österreichische Wasser- und Abfallwirtschaft, 11-12/2017. https://doi.org/10.1007/ s00506-017-0420- 1

BMLFUW (Bundesministerium für Land- und Forstwirtschaft, Umwelt und Wasserwirtschaft) (2010): BGBl. II Nr. 476/2010 - Verordnung über die Verbrennung von Abfällen (Abfallverbrennungsverordnung - AVV). Wien, Österreich

BMLFUW (Bundesministerium für Land- und Forstwirtschaft, Umwelt und Wasserwirtschaft) (1996): BGBl. Nr. 186/1996 - Verordnung über die allgemeine Begrenzung von Abwasseremissionen in Fließgewässer und öffentliche Kanalisationen (Abwasseremissionsverordnung - AAEV). Wien, Österreich

BMLFUW (Bundesministerium für Land- und Forst-, Umwelt und Wasserwirtschaft) (2015): Die Bestandsaufnahme der Abfallwirtschaft in Ö., Statusbericht 2015. Vienna, Austria

Cencic, O. \& Rechberger, H., (2008): Material

Flow analysis with software STAN. J. Environ.

Eng. Manage. 18 (1), 3-7
Deditz, J., Pinkel, M. \& Pomberger, R. (2014): Concepts for Processing Solid Recovered Fuels of Different Waste Origins for Waste-to-Energy Plants. In: Thomé-Kozmiensky, K. \& Thiel, S. (Hrsg.): Waste Management, Band 4. Wasteto-Energy. Neuruppin: TK Verlag Karl ThoméKozmiensky, 2014. ISBN: 978-3-944310-15-2 EK (Europäische Kommission) (2015a): Closing the loop: Commission adopts ambitious new Circular Economy Package to boost competitivenesss, create jobs and generate sustainable growth. Brüssel, Belgien

EK (Europäische Kommission) (2015b): Proposal for the Directive 94/62/EG of the European Parliament and of the Council 5 Dezember 2015 on packaging and packaging waste. Brüssel, Belgien

EK (Europäische Kommission) (2015c): Proposal for the Directive 2008/98/EC of the European Parliament and of the Council 5 Dezember 2015on waste. Brüssel, Belgien

EK (Europäische Kommission) (2017a): Proposal for a Directive of the European Parliament and of the Council amending Directive 94/62/EC on packaging and packaging waste - Outcome of the European Parliament's first reading (Strasbourg, 13 to 16 March 2017). Brüssel, Belgien

EK (Europäische Kommission) (2017b): Proposal for a Directive of the European Parliament and of the Council amending Directive 2008/98/EC on waste - Outcome of the European Parliament's first reading (Strasbourg, 13 to 16 March 2017). Brüssel, Belgien

EPA (United States Environmental Protection Agency) (2016): Advancing Sustainable Materi- als Management - Assessing Trends in Material Generation, Recycling, Composting, Combustion with Energy Recovery and Landfilling in the United States. https://www.epa.gov/sites/ production/files/2016-11/documents/2014 smmfactsheet_508.pdf. Gesehen 3. März 2017

Kranzinger, L., Pomberger, R., Schwabl, D. \& Bauer, M. (2016): Quo vadis Kunststoffrecycling - Bestandsaufnahme der polyolefinen Kunststoffe in der österreichischen Abfallwirtschaft In: R. Pomberger et al. (Hrsg.): RecyDepoTech 2016. Tagungsband, Leoben, 8-11 November, pp. 583-588. Lavanttal, Österreich: Christian Theiss GmbH. ISBN: 978-3-200-04777-8

N. N. (2000): Abbaubarkeit. Wasser-Wissen, Institut für Umweltverfahrenstechnik- Universität Bremen, 2000 http://www.wasserwissen.de/ abwasserlexikon/a/abbaubarkeit.htm. Gesehen 9. Juli 2017

Plastic (Plastic Insights) (2017): Global Consumption of Plastic Materials By Region (1980-2015). https://www.plasticsinsight.com/ global-consumption-plastic-materials-region1980-2015/. Gesehen 10. Juli 2017

Plastics (Plastics Europe) (2015a): Plastics-The Facts 2015; An Analysis of European plastics production, demand and waste data. Brüssel, Belgie: Plastics Europe. http://www.plasticseurope.org/ Document/plastics-the-facts-2015.aspx. Gesehen 12. November 2016

Plastics (Plastics Europe) (2015b): Post-Consumer Plastic Waste Management in European Countries 2014 - Final Report. Brüssel, Belgien

Plastics (Plastics Europe) (2017): Plastics - The Facts 2016; An Analysis of European plastics pro- 
duction, demand and waste data. Brüssel, Belgie: Plastics Europe. http://www.plasticseurope.org/ Document/plastics-the-facts-2016.aspx. Gesehen 12. November 2016

PWMI (Plastic Waste Management Institute Japan) (2015): Plastic Products, Plastic Waste and Resource Recovery - Background information and notes on the publication of the Flowchart of Plastic Products, Plastic Waste and Resource
Recovery. http://www.pwmi.or.jp/ei/siryo/ei/ ei_pdf/ei46.pdf. Gesehen 8. Juli 2017

PWMI (Plastic Waste Management Institute Japan) (2016): An Introduction to Plastic Recycling. http://www.pwmi.or.jp/ei/plastic _ recycling_2016.pdf. Gesehen 8. Juli 2017

Sarc, R. \& Lorber, K.E. (2013): Production, quality and quality assurance of Refuse Derived Fuels
(RDFs). In: Waste Management 33 (2013). ISSN: 0956-053X. S. 1825-1834

Sarc, R., Lorber, K.E., Pomberger, R., Rogetzer, M. \& Sipple, E.M. (2014): Design, Quality and Quality Assurance of Solid Recovered Fuels (SRF) for the Substitution of Fossil Feedstock in the Cement Industry. In: Waste Management \& Research 32 (7): 565-585 